\title{
Outcomes following Suboccipital Decompressive Craniectomy for Posterior Fossa Stroke with Malignant Cerebellar Oedema: A Case Series and Review of the Literature
}

\author{
Michael Brooks, MD1,2*, Rebecca Nguyen, MD², Ganeshwaran Shivapathasundram, MBBS, \\ FRACS $^{1}$ and Mark Sheridan, MBBS, FRACS ${ }^{1}$
}

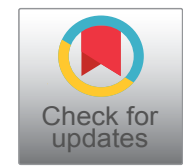

${ }^{1}$ Department of Neurosurgery, Liverpool Hospital, New South Wales, Australia

${ }^{2}$ South West Sydney Clinical School, School of Medicine, University of New South Wales (UNSW), New South Wales, Australia

*Corresponding author: Dr. Michael Brooks, Department of Neurosurgery, South West Sydney Clinical School, School of Medicine, University of New South Wales, Randwick, New South Wales, Australia; Department of Neurosurgery, Liverpool Hospital, 1 Elizabeth St, Liverpool, NSW, 2170, Australia, Tel: +614-151-84123, Fax: +612-8738-3343

\begin{abstract}
Background: Suboccipital decompressive craniectomy is a procedure that has been performed to treat malignant cerebellar oedema secondary to posterior fossa strokes. Due to the rarity of the procedure, more evidence is required to better identify factors associated with good or poor outcomes to aid in appropriate patient selection for surgical intervention. Currently known good prognostic factors include reduced time until surgery from deterioration and unilateral infarction.
\end{abstract}

Method: A retrospective review was performed to identify all patients who underwent a suboccipital decompressive craniectomy at a major tertiary hospital in Sydney, Australia over a ten-year period. Outcomes were recorded for a oneyear follow up period, where a good functional outcome was defined as a modified Rankin Scale score of two or less. Other data collected included patient factors and surgical factors.

Results: A total of seven patients were identified who underwent suboccipital decompressive craniectomy for cerebellar oedema secondary to an ischaemic stroke. Of these patients, three died and the remaining four had good long-term functional outcome. A variety of factors were identified which were more common in patients with a positive functional outcome at one-year follow-up, including debridement of surgical tissue, and younger age. Negative factors we noted included bilateral infarction, brainstem infarction, and a delay from the time of deterioration until surgery.
Conclusion: Suboccipital decompressive craniectomy remains a valid procedure for malignant cerebellar oedema secondary to posterior fossa stroke, and further identification of factors which are associated with a good outcome can help with patient selection.

\section{Keywords}

Suboccipital craniectomy, Decompressive craniectomy, Outcomes, Stroke, Cerebellar oedema

\section{Introduction}

Malignant cerebellar oedema occurring secondary to ischaemic strokes of the posterior fossa is a major cause of mortality due to brainstem infarction or mass effect resulting in hydrocephalus, tonsillar herniation, and brainstem compression. Best treatment requires both medical and surgical management. Surgical management includes insertion of external ventricular drain (EVD) with suboccipital decompressive craniectomy (SDC) [1]. Medical management comprises a number of measures including elevating the head of the bed, blood pressure control and use of neuroprotective measures such as maintaining normothermia [2] and normocapnia [3] to control intracranial pressure. While the role of a decompressive craniectomy is well reported for malignant oedema in middle cerebral artery (MCA)

Citation: Brooks M, Nguyen R, Shivapathasundram G, Sheridan M (2021) Outcomes following Suboccipital Decompressive Craniectomy for Posterior Fossa Stroke with Malignant Cerebellar Oedema: A Case Series and Review of the Literature. Neurosurg Cases Rev 4:083. doi. org/10.23937/2643-4474/1710083

Accepted: October 07, 2021; Published: October 09, 2021

Copyright: (c) 2021 Brooks M, et al. This is an open-access article distributed under the terms of the Creative Commons Attribution License, which permits unrestricted use, distribution, and reproduction in any medium, provided the original author and source are credited. 

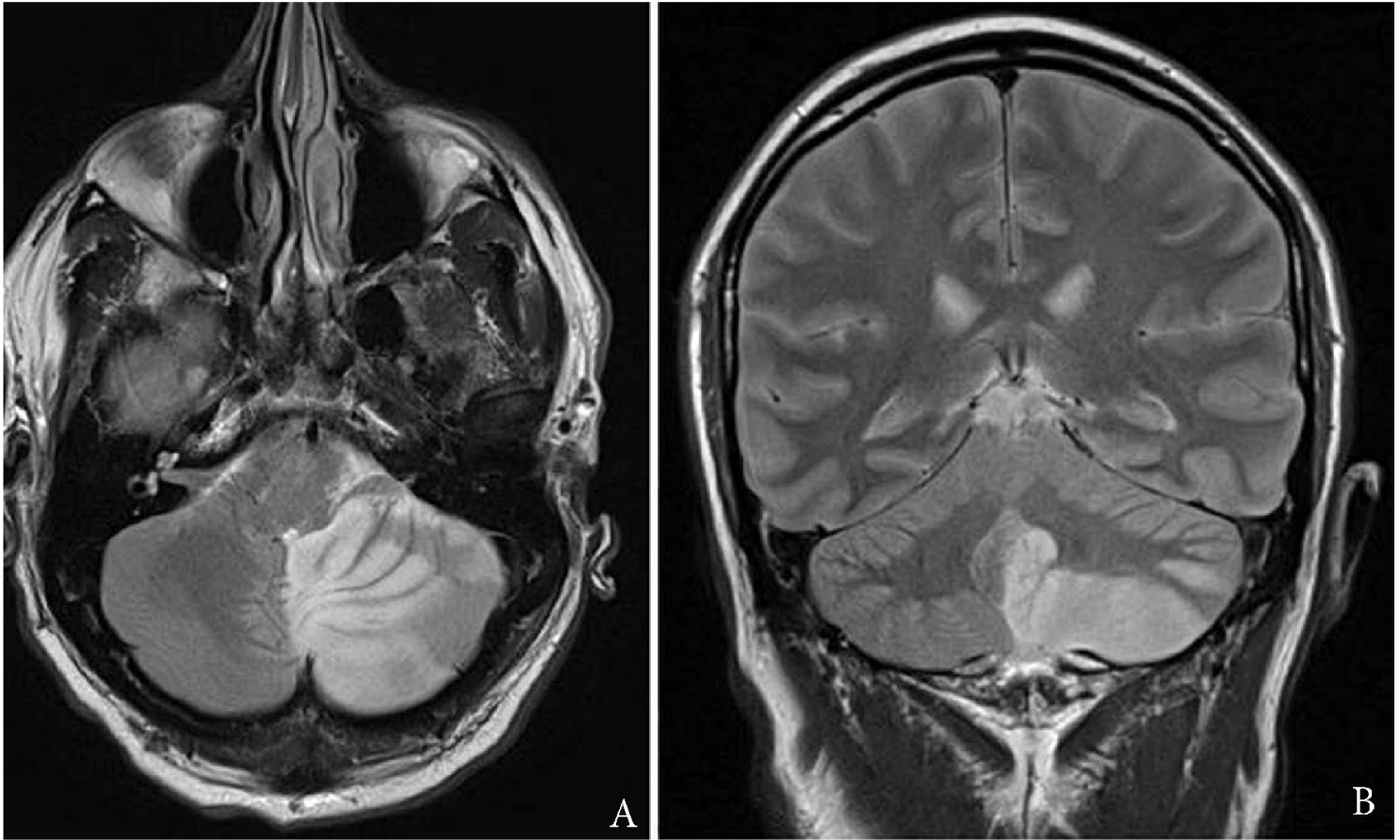

Figure 1: A) T2-weighted MRI axial slice demonstrating left-sided inferior cerebellar infarction; B) Corresponding T2-weighted MRI coronal slice.

territory ischaemic strokes [1,4-7], there is a paucity of high-quality evidence on the outcomes following SDC.

SDC has been reported in the literature since as early as $1956[8,9]$, with subsequent work by Lehrich, et al. [10] in 1970 further demonstrating the potential role in mortality reduction. Current evidence is still limited to retrospective case series $[11,12]$, with no randomised control trials available. A recent meta-analysis, published in 2017, identified eleven observational studies with 283 patients and found that SDC has benefit as a lifesaving procedure, but patient selection based on certain factors such as age and unilateral infarction could further improve outcomes, however further evidence is still required [13]. We present a retrospective review of seven consecutive cases that presented to Liverpool Hospital over a ten-year period.

\section{Methods}

Ethics approval was obtained allowing for a retrospective review of all patients undergoing an SDC at Liverpool Hospital, a major trauma centre with a neurosurgical unit in Sydney, Australia, between January 1, 2010 and December 31, 2019. A total of sixteen participants aged between 18 and 64 were identified. Inclusion criteria were patients aged over 18 who underwent SDC for ischaemic cerebellar stroke. Patients were excluded if they underwent SDC for a different indication. Of these sixteen, seven (44\%) met our inclusion criteria of undergoing emergent SDC for cerebellar oedema secondary to an ischaemic stroke. The primary outcome assessed was modified Rankin scale [14] (mRS) on discharge from our facility and/or from rehabilitation, where a score of 0-2 was considered a positive functional outcome [15]. Secondary outcomes included mortality, mRS at one-year follow up, and admission to rehabilitation facility on discharge. Data were also collected for glasgow coma scale (GCS) on admission and pre-operatively, placement on an external ventricular drain (EVD), debridement of infarcted tissue, time from ictus to surgery, and admission to rehabilitation. Follow-up was obtained to the maximum possible duration in each case.

\section{Case Series}

\section{Case 1}

A 47-year-old male presented with sudden onset of severe dizziness, nausea and vomiting, and profound ataxia. Initial MRI brain demonstrated a hyperintense lesion on the left side of the medulla secondary to infarcts in the left posterior inferior cerebellar artery (PICA) and anterior inferior cerebellar artery (AICA) territories, and a thrombosed aneurysm of the left vertebral artery (Figure 1). He was GCS 15 on arrival and admitted to the ward. Three days later he had a sudden drop in GCS to 10. Urgent CT brain demonstrated a hypodense left cerebellar lesion and compression of the fourth ventricle with tonsillar herniation. Subsequently, he underwent an urgent SDC with EVD insertion and resection of necrotic cerebellar tissue.

The patient spent 3 days in the intensive care unit (ICU) before being transferred to the ward. He was discharged to rehabilitation hospital after 21 days, where he remained for a further 3 days. At the time of discharge from the acute facility, he required mild assistance with mobilisation due to an ataxic gait (mRS3). His gait continued to improve while in the rehabilitation 
hospital with no assistance required despite persistent ataxia (mRS 1). On review 1 year post-operatively, he was independent for mobility and in self-care activities of daily living ( $\mathrm{mRS} 0$ ).

\section{Case 2}

A 64-year-old female presented to a peripheral hospital with a 6-week history of generalised fatigue and lethargy. CT brain demonstrated a small area of hypodensity in the right cerebellum. Two days later, she developed an episode of impaired gaze, with no other neurological deficits. However, no repeat imaging was performed at this time. A further two days later, she experienced a sudden drop in GCS to 8 associated with slurred speech, worsening diplopia, confusion, and sluggish pupillary light reflex. A repeat CT brain demonstrated progression of the cerebellar hypodensity and crowding of the foramen magnum. She was transferred to Liverpool Hospital and underwent urgent SDC.

She remained in ICU for 7 days, and demonstrated some improvement in GCS, however she never recovered to a point where she was safe to extubate, and repeat imaging demonstrated a progression of her infarction. She began to deteriorate, and the decision was made to transition to palliative treatment with a one-way extubation before she passed away.

\section{Case 3}

A 52-year-old male presented with a prodrome of vertigo, ataxia, headache and vomiting. He was initially discharged without imaging, but developed a worsening headache, agitation and dysphagia. On readmission, he was GCS 14 and otherwise neurologically intact. CT brain demonstrated a hypodensity involving the left-sided posterior surface of the cerebellum consistent with an ischaemic stroke and moderate hydrocephalus. He deteriorated to GCS 8 and was intubated. He underwent urgent SDC and EVD insertion.

He remained an inpatient for 26 days, with a prolonged stay due to ongoing inpatient rehabilitation. His functional status improved considerably, requiring moderate assistance on discharge from our facility (mRS 2) and he was transferred to a specialist rehabilitation hospital for a further 5 days due to a persistent ataxic gait. On discharge from rehabilitation, he was asymptomatic and had returned to his premorbid state (mRS 0). He was able to return to work in full capacity 3 months after his surgery. On follow-up one year later, no change to his condition was noted.

\section{Case 4}

A 33-year-old female was urgently transferred to our facility with a decreased level of consciousness to GCS 7 following presentation with a headache, confusion and hypertension three days earlier. CT brain demonstrated a left-sided PICA infarct with obstructive hydrocephalus and effacement of the fourth ventricle. She underwent an emergent SDC and placement on an EVD, and due to the degree of intraoperative raised intracranial pressure, an area of infarcted cerebellum was resected.

She remained in intensive care for 2 days, before being transferred to the ward. Her admission was complicated by acute-on-chronic renal failure. She remained an inpatient for a total of 15 days before being transferred to a specialist rehabilitation hospital. On admission to rehabilitation, she required minimal assistance with mobility, but was independent with self-cares such as dressing and toileting, (mRS 3). She remained in rehabilitation for 20 days where she was able to return to her pre-morbid functional state and was discharged with no ongoing deficits (mRS 0 ). No neurological deficits remained at 1-year follow-up. Unfortunately, almost 2 years later, she suffered a spontaneous brainstem haemorrhage and passed away.

\section{Case 5}

A 58-year-old female presented to hospital with an acute onset occipital headache and persistent vertiginous symptoms. CT brain demonstrated a subacute left PICA territory infarct, with mild local mass effect and no haemorrhagic transformation. Three days later, she developed a sudden drop in GCS to 13, associated with new bilateral upper and lower limb weakness. Repeat CT scan demonstrated progression of the posterior fossa infarct, with a moderate sized area of infarction located within the inferior left cerebellum and evidence of tonsillar herniation. She was urgently transferred to our facility for an urgent SDC and insertion of right frontal EVD. She remained drowsy postoperatively, with fluctuating levels of consciousness however was initially extubated day 1 post-operative. On day 5 post-operatively her GCS dropped to 4, requiring re-intubation. No acute cause was identified on repeat imaging, MRI brain demonstrated small foci of further infarction in the midbrain and right cerebellar hemisphere. In spite of this, she made a good neurological recovery and was extubated after nine days. She remained in ICU for a total of 15 days before discharge back to the ward.

She was discharged to rehabilitation facility after 5 days on the ward. At the time of transfer, she was requiring moderate assistance with mobility and activities of daily living ( $m R S 4)$. She remained in rehabilitation for 2 months, where she was discharged able to mobilise with stand by assistance and no aids (mRS 2). At 1-year follow-up, she had persistent leftsided hemiplegic gait but remained independent of mobility (mRS 1).

\section{Case 6}

A 51-year-old male was transferred to Liverpool Hospital after presenting with dizziness, headache, right facial droop, left upper and lower limb weakness, 

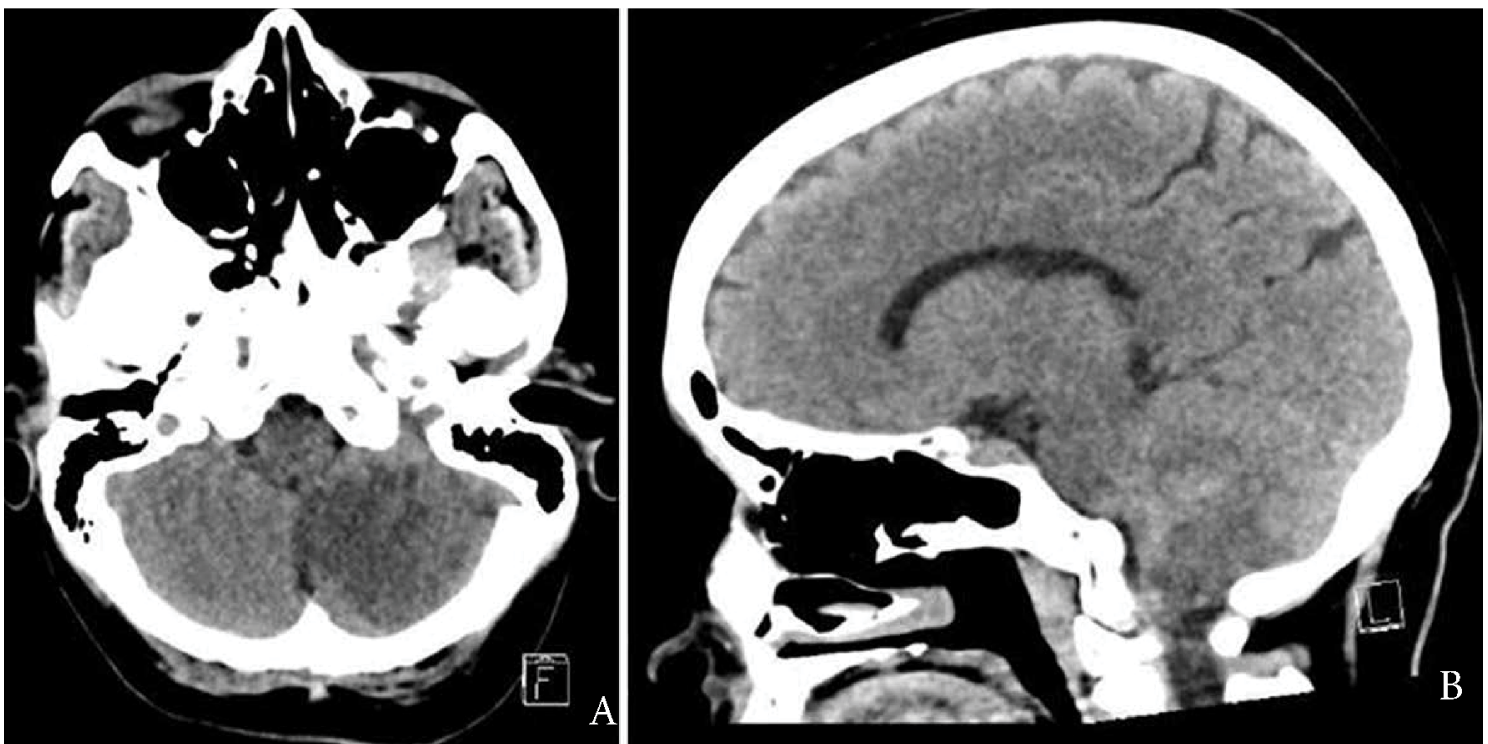

Figure 2: A) Axial slice of CT demonstrating left cerebellar ischaemic stroke with associated oedema; B) Corresponding saggital CT.
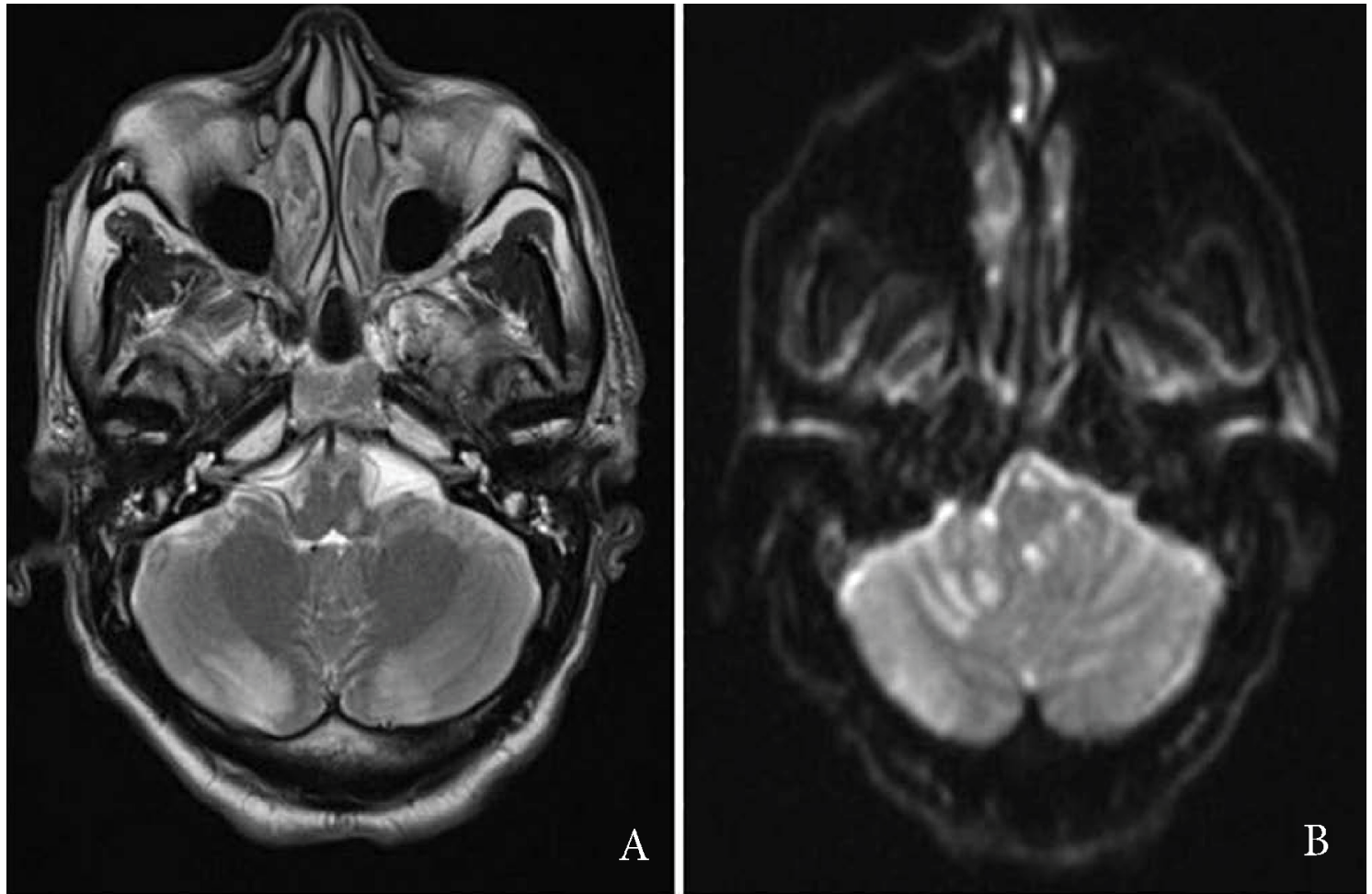

Figure 3: A) T2-weighted MRI axial slice demonstrating bilateral cerebellar ischaemia with associated oedema; B) MRI diffusion-weighted imaging demonstrating areas of acute infarction bilateral areas of infarction.

and slurred speech. CT brain demonstrated an acute left superior cerebellar hemisphere infarct with an approximately $7 \mathrm{~mm}$ distal basilar artery occlusion but normal flow of posterior cerebral arteries bilaterally. He underwent successful clot retrieval of basilar and vertebral artery occlusion (Figure 2). However, in recovery, he became agitated and developed right sided facial droop and right sided weakness, and his GCS subsequently reduced to 10 (E2V2M6). He required emergent intubation and repeat $\mathrm{CT}$ demonstrated acute bilateral cerebellar infarct associated with 8 $\mathrm{mm}$ tonsillar herniation, effacement of the fourth ventricle and basal cisterns, with mild hydrocephalus. Additionally, there was evidence of acute haemorrhage in the left cerebellar hemisphere and an acute right middle cerebral artery territory infarct.

The patient subsequently underwent emergent SDC with C1 posterior arch resection and EVD insertion, approximately 10 hours after initial hospital presentation. Post-operatively, the patient remained GCS3 after sedation was ceased with absent brainstem reflexes. Circulatory death was diagnosed 6 days 
later and the patient was referred for organ donation following discussion with family.

\section{Case 7}

A 54-year-old male presented following a one-day history of progressive hearing loss, gait disturbance and vomiting. He was found with a reduced GCS of 5 the next day, and taken urgently to hospital, however his GCS recovered spontaneously en route to 12 . He deteriorated with pupils becoming unequal and sluggish to respond while in the emergency department and was subsequently intubated. MRI brain demonstrated a large area of infarction in the PCA territory, as well as both superior cerebellar artery territories, with no distal flow visible (Figure 3). He underwent urgent clot retrieval, wherein the $\mathrm{P} 2$ segment on the left posterior cerebral artery (PCA) was sacrificed due to intractable haemorrhage. He underwent an urgent SDC, however no meaningful neurological recovery was demonstrated with persistently absent brainstem reflexes and reduced GCS, and he passed away the next day.

\section{Discussion}

We have described the outcomes of seven consecutive patients who underwent SDC at Liverpool Hospital. All cases underwent SDC for acute posterior fossa circulation ischaemia with associated malignant oedema. In all cases, the surgery occurred within 80 hours following the initial infarct, and all operations occurred within twenty-four hours of clinical deterioration. Of the seven cases, three passed away, two had a poor neurological recovery on discharge from our facility, and the final two had meaningful neurological recovery on discharge. On discharge from the rehabilitation hospital, all surviving patients demonstrated meaningful neurological recovery $(\mathrm{mRS} \leq 2)$. These results are similar to the reported rates in the literature, with a combined poor outcome (defined as $\mathrm{mRS} \geq 3$ on discharge) of $48 \%$ [13]. In considering results at one year follow up, all surviving patients demonstrated good functional recovery and independence at this time.

Participant characteristics have been reported in Table 1. The average age of participants was 59 years old, with a range of 33-64 years. The most common comorbidity in patients was hypertension. Follow up was obtained to at least one year for all surviving patients. The most common arterial territory was the left PICA. GCS on admission ranged from 12-15, with a median of 15. Surgical factors are reported in Table 2. Brainstem infarction occurred in two patients, and three patients had bilateral cerebellar infarcts. GCS pre-operatively ranged from 3-13. Time from onset to surgery ranged from 13 to 76 hours, and time from deterioration to surgery from 2 to 16 hours.

The impact of certain intra-operative factors such as EVD insertion and resection of infarcted tissue intraoperatively remains inconclusive in current literature
[16]. In this series, EVD insertion was considered a routine aspect of the procedure and resection of infarcted tissue was done on an as needed basis as per the current procedural standard. Cerebellar resection occurred in two of the patients, one of whom had a favourable recovery at discharge, and both had a favourable outcome on long-term follow-up. This agrees with the previously reported outcomes stating resection of necrotic tissue as a valid adjunct to SDC to reduce mass effect and allow for further expansion of the oedema $[13,16,17]$.

The impact of bilateral lesions on functional outcomes and survival still remains to be clarified. Owing to the rarity of bilateral infarction, very little data is available to guide clinicians in the management [18]. In our series, three patients were identified with a bilateral infarction and two were associated with death. Contrary to the series by Tsitsopoulos, et al. [18], our series noted poor outcome with bilateral infarction. Previous literature has supported SDC in bilateral cerebellar stroke as a valid life-saving procedure [17], however we would argue against this. Bilateral strokes demonstrate progressively worse pathology with a larger degree of oedema and poor prognosis in both the patients in this series.

Current evidence supports early neurological recovery as a positive outcome. In case five, early neurological deterioration was noted post-operatively, yet good outcome was obtained. In case five, a drop in GCS was noted and urgent re-intubation and sedation was required with progression of the infarct to the right cerebellar hemisphere noted. Contrary to past literature [19], this case demonstrates that poor early neurological recovery and deterioration do not necessarily correlate with long-term functional outcome.

Infarcts associated with brainstem infarction have typically been noted to have a worse survival than those without brainstem involvement [20]. This is largely attributed to eloquence of brainstem and the significant impairment that arises with brainstem infarction. In our series, two patients had associated brainstem infarctions, and both patients died prior to discharge. This supports current literature where brainstem infarction has noted a worse outcome and could be used as a discriminatory factor in the decision on whether to operate.

Time from stroke onset to surgery and time from deterioration to surgery have both been considered as factors which may influence outcome [20]. All of our patients were operated on within 80 hours of the ictus, and within twenty-four hours of clinical deterioration. In our group, early surgery did not lead to better outcomes, noting that the two patients who underwent SDC within the first 24 hours since symptom onset had a poor outcome. However, we noted better functional outcomes when considering the time from 

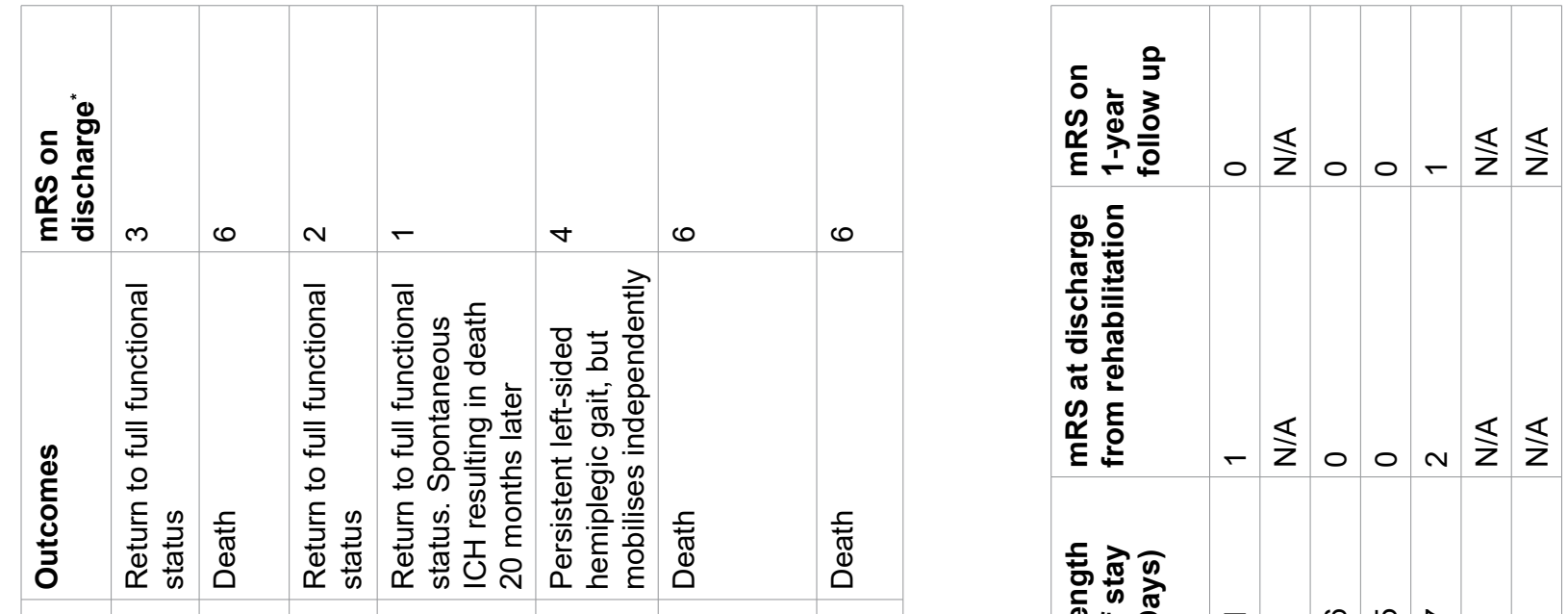

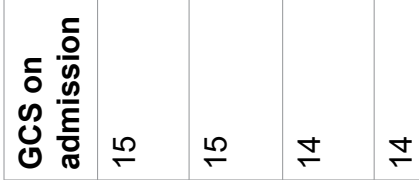

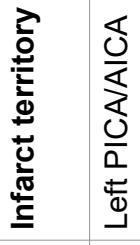

$\stackrel{\llcorner}{\circ} \stackrel{\circ}{\circ}$

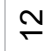

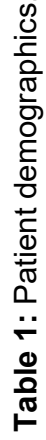
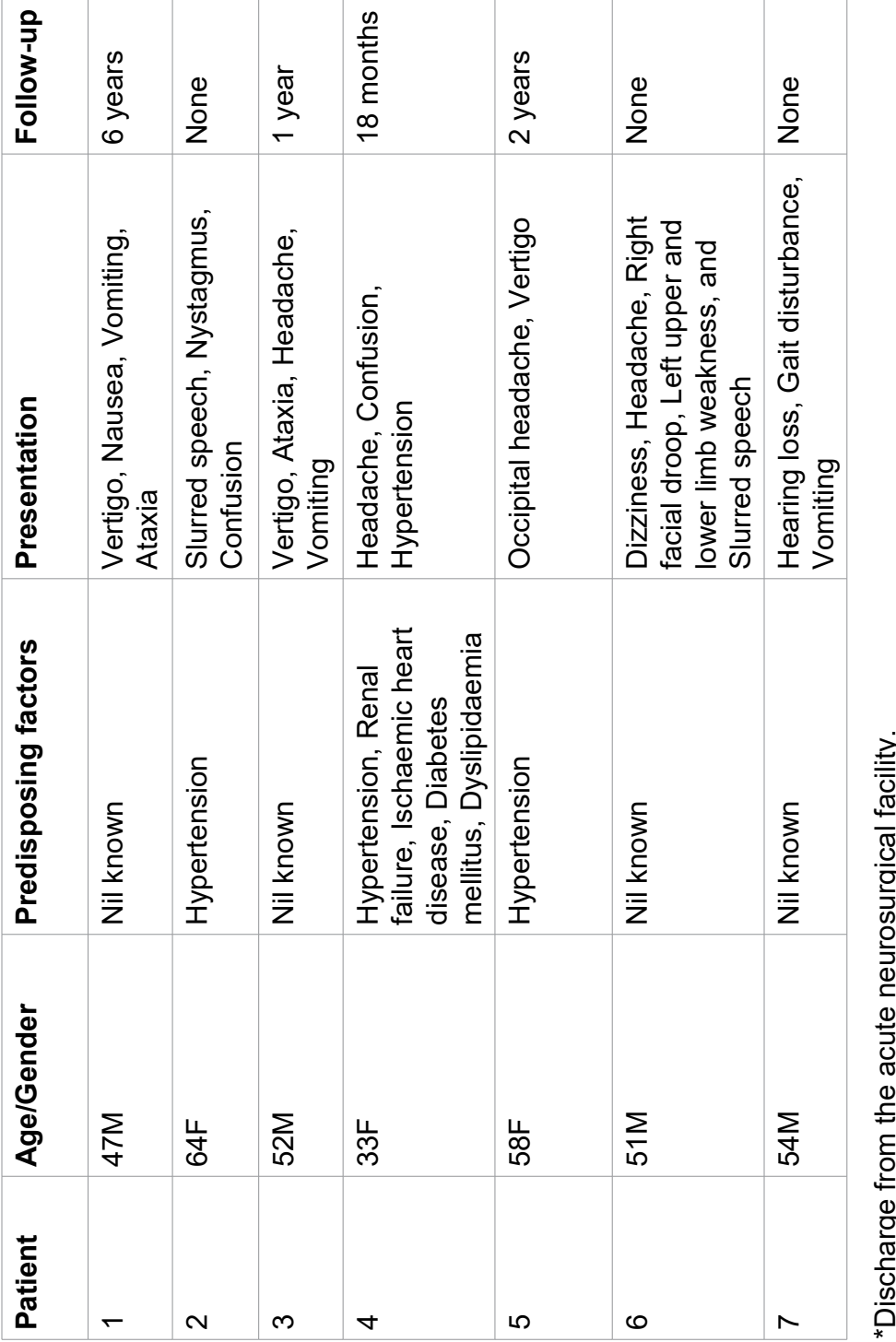

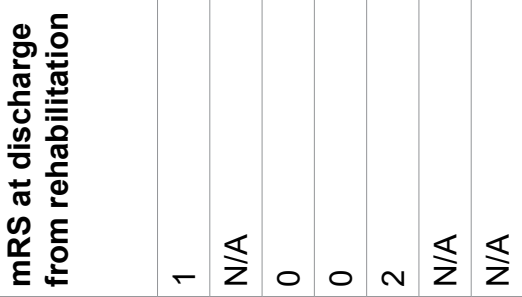

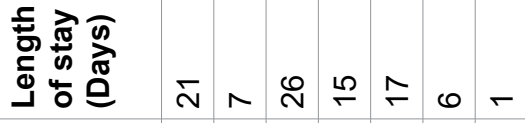
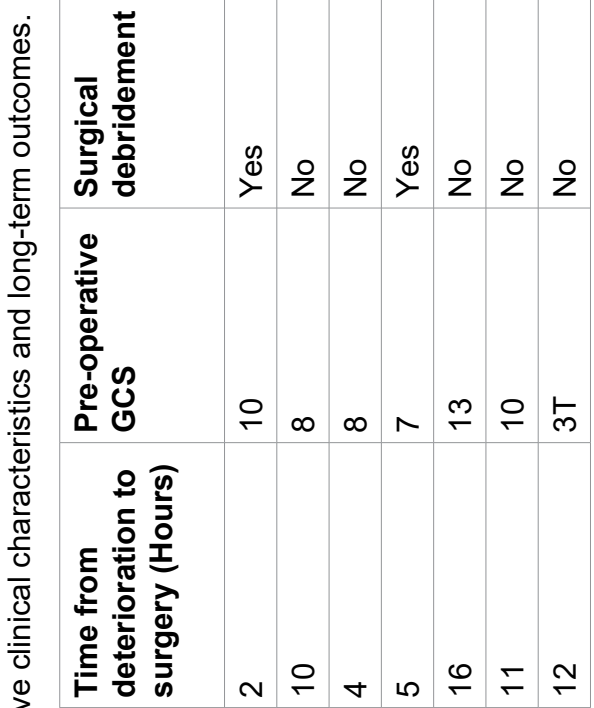

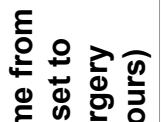

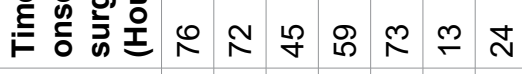

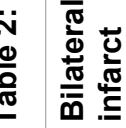

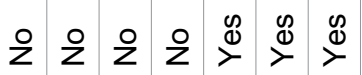

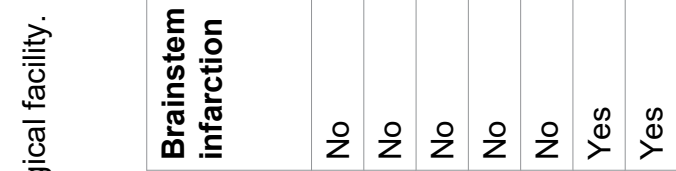

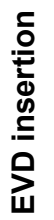

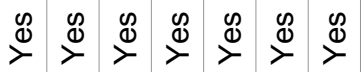

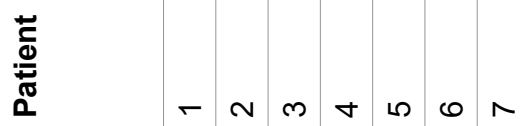


further clinical deterioration to surgery. Here, all patients who underwent SDC in under 10 hours of acute deterioration had a good outcome compared to one out of the four who were operated on in ten hours or more. This advocates for continual monitoring post ictus and urgent action when clinical deterioration occurs.

Other factors have been implicated in poor functional outcome such as age, GCS on admission and preoperative GCS. Both patients under 50-years-old had a positive outcome on discharge from rehabilitation, however no link was able to be made with patient age and outcome. Further to this, no link was noted between both GCS on admission and pre-operative GCS with either good or poor outcome. In both patients who had superior cerebellar artery strokes, poor outcome was noted, however these patients also suffered from bilateral strokes and as such poor outcome is attributed to the bilateral stroke rather than the arterial territory.

\section{Conclusion}

This series supports the idea that SDC can be used as a life-saving measure for malignant cerebellar oedema secondary to ischaemic stroke. In all patients who survived the initial admission, positive outcomes were noted at 1-year follow-up. Factors associated with a worse outcome include bilateral infarctions, brainstem infarctions and a delay from clinical deterioration to surgery. SDC remains a life-saving procedure for malignant cerebellar oedema secondary to ischaemic stroke, however patient selection is important.

\section{Authors Declaration}

No funding was received for this review. The authors report no conflict of interest concerning the materials or methods used in this study or the findings specified in this paper. Ethics approval for this study was obtained from South West Sydney Local Health District Human Research Ethics Committee, approval number 2020/ ETH01493. All authors contributed equally to the project.

\section{References}

1. Agarwalla PK, Stapleton CJ, Ogilvy CS (2014) Craniectomy in acute ischemic stroke. Neurosurgery 74: S151-S162.

2. Fox JL, Vu EN, Doyle-Waters M, Brubacher JR, AbuLabanet R, et al. (2010) Prophylactic hypothermia for traumatic brain injury: A quantitative systematic review. CJEM 12: 355-364.

3. Doppmann P, Meuli L, Sollid SJ, Filipovic M, Knapp J, et al. (2021) End-tidal to arterial carbon dioxide gradient is associated with increased mortality in patients with traumatic brain injury: A retrospective observational study. Scientific reports 11: 1-9.

4. Rahmig J, Wopking S, Juttler E, Uhlmann L, Limprecht R, et al. (2019) Decompressive hemicraniectomy in elderly patients with space-occupying infarction (DECAP): A prospective observational study. Neurocrit Care 31: 97106.
5. Juttler E, Bosel J, Amiri $H$, Schiller $P$, Limprecht $R$, et al. (2011) DESTINY II: Decompressive surgery for the treatment of malignant infarction of the middle cerebral arterY II. Int J Stroke 6: 79-86.

6. Cruz-Flores S, Berge E, Whittle IR (2012) Surgical decompression for cerebral oedema in acute ischaemic stroke. Cochrane Database Syst Rev 1: CD003435.

7. Vahedi K, Hofmeijer J, Juettler E, Vicaut E, George B, et al. (2007) Early decompressive surgery in malignant infarction of the middle cerebral artery: A pooled analysis of three randomised controlled trials. Lancet Neurol 6: 215-222.

8. Fairburn B, Oliver LC (1956) Cerebellar softening. Br Med J 1: 1335.

9. Lindgren SO (1956) Infarctions simulating brain tumours in the posterior fossa. J Neurosurg 13: 575-581.

10. Lehrich JR, Winkler GF, Ojemann R (1970) Cerebellar infarction with brain stem compression: Diagnosis and surgical treatment. Arch Neurol 22: 490-498.

11. Kim MJ, Park SK, Song J, Oh SY, Lim YC, et al. (2016) Preventive suboccipital decompressive craniectomy for cerebellar infarction: A retrospective-matched case-control study. Stroke 47: 2565-2573.

12. Juttler E, Schweickert S, Ringleb PA, Huttner HB, Kohrmann $M$, et al. (2009) Long-term outcome after surgical treatment for space-occupying cerebellar infarction: Experience in 56 patients. Stroke 40: 3060-3066.

13. Ayling OGS, Alotaibi NM, Wang JZ, Fatehi M, Ibrahim GM, et al. (2018) Suboccipital decompressive craniectomy for cerebellar infarction: A systematic review and metaanalysis. World Neurosurg 110: 450-459.

14. Van Swieten J, Koudstaal P, Visser M, Schouten HJ, Gijn JV, et al. (1988) Interobserver agreement for the assessment of handicap in stroke patients. Stroke 19: 604-607.

15. Bonita R, Beaglehole R (1988) Recovery of motor function after stroke. Stroke 19: 1497-1500.

16. Tartara F, Bongetta D, Colombo EV, Bortolotti C, Cenzato M, et al. (2018) Strokectomy and extensive cerebrospinal fluid drainage for the treatment of space-occupying cerebellar ischemic stroke. World Neurosurg 115: e80-e84.

17. Pfefferkorn T, Eppinger U, Linn J, Birnbaum T, Herzog $\mathrm{J}$, et al. (2009) Long-term outcome after suboccipital decompressive craniectomy for malignant cerebellar infarction. Stroke 40: 3045-3050.

18. Tsitsopoulos PP, Tobieson L, Enblad P, Marklund N (2011) Clinical outcome following surgical treatment for bilateral cerebellar infarction. Acta Neurol Scand 123: 345-351.

19. Tsitsopoulos PP, Tobieson L, Enblad P, Marklund N (2011) Surgical treatment of patients with unilateral cerebellar infarcts: Clinical outcome and prognostic factors. Acta Neurochir (Wien) 153: 2075-2083.

20. Lindeskog D, Lilja-Cyron A, Kelsen J, Juhler M (2019) Longterm functional outcome after decompressive suboccipital craniectomy for space-occupying cerebellar infarction. Clin Neurol Neurosurg 176: 47-52. 